\section{Alphablocker bei Urolithiasis doch gerechtfertigt?}

\author{
Die Therapie der Urolithiasis mit Alphablockern wurde 2015 von den Autoren \\ einer randomisierten Studie infrage gestellt. Jetzt halten die Autoren einer \\ aktuellen Metaanalyse dagegen: Die Wirksamkeit sei klar gegeben, vor allem \\ bei Patienten mit größeren Steinen.
}

$\mathrm{D}_{\mathrm{s}}$ ie Ergebnisse der randomisierten Studie von Prof. Robert Pickard, Newcastle University, England, und seinen Kollegen, publiziert im Mai $2015 \mathrm{im}$ Fachblatt The Lancet, haben eine seit Jahren schwelende Diskussion befeuert: Wie sinnvoll ist die medikamentös expulsive Therapie (MET) bei Patienten mit einer Harnleiterkolik auf der Basis einer primär nicht interventionsbedürftigen Urolithiasis? Die aktuelle deutsche Leitlinie sieht für neu diagnostizierte Harnleiterkoliken den Off-Label-Einsatz von Alphablockern im Rahmen der MET als Kann-Empfehlung vor, wenngleich ihr Nutzen bis dato lediglich durch kleinere Studien minderer Qualität belegt war.

Pickard und sein Team hatten die Frage in einem randomisierten kontrollierten Design mit über 11.000 Patienten untersucht und waren zu dem Schluss gekommen, dass weder der Alphablocker Tamsulosin noch der Kalziumantagonist Nifedipin bei dieser Indikation wirksamer seien als ein Scheinmedikament. Dem widerspricht nun das Team um Dr. John M. Hollingsworth von der University of Michigan in Ann Arbor, USA: In ihrer in der Fachzeitschrift The British Medical Journal (BMJ) publizierten Metaanalyse liefern die US-Forscher Belege dafür, dass Alphablocker dazu beitragen, die Passage zumindest größerer Harnlei-

tersteine zu erleichtern, und zwar unabhängig von deren Lokalisation.

Grundlage der Metaanalyse waren 55 randomisierte, kontrollierte Studien, in denen jeweils Alphablocker als Haupttherapeutikum einem Placebo gegenübergestellt wurden. Neben den in zwei Cochrane-Reviews von 2012 und 2014 bereits berücksichtigten RCT (,randomized controlled trials") umfasst die aktuelle Metaanalyse zahlreiche weitere, darunter auch die besagte Studie von Pickard et al. Insgesamt wurden in der US-Metaanalyse die Daten von 5.990 Patienten mit Harnleitersteinen ausgewertet. Während Pickard et al. jedoch den Anteil von Patienten ermittelt hatten, bei denen innerhalb eines Monats nach Randomisierung keine weitere Intervention zur Steinentfernung erforderlich wurde, wählten die US-Forscher als primären Endpunkt den Anteil der Patienten mit dokumentiertem Steinabgang.

Wie die gepoolte Analyse zeigte, lag die Wahrscheinlichkeit eines Steinabgangs unter einer Alphablockertherapie um 49 \% höher als in der Placebogruppe. Die Qualität der ausgewerteten Studien wurde insgesamt als „mittelmäßig“ beurteilt. Die gepoolte Risikodifferenz betrug laut Hollingsworth und Kollegen 0,27 , das heißt, es müssten vier Patienten behandelt werden, damit einer von der

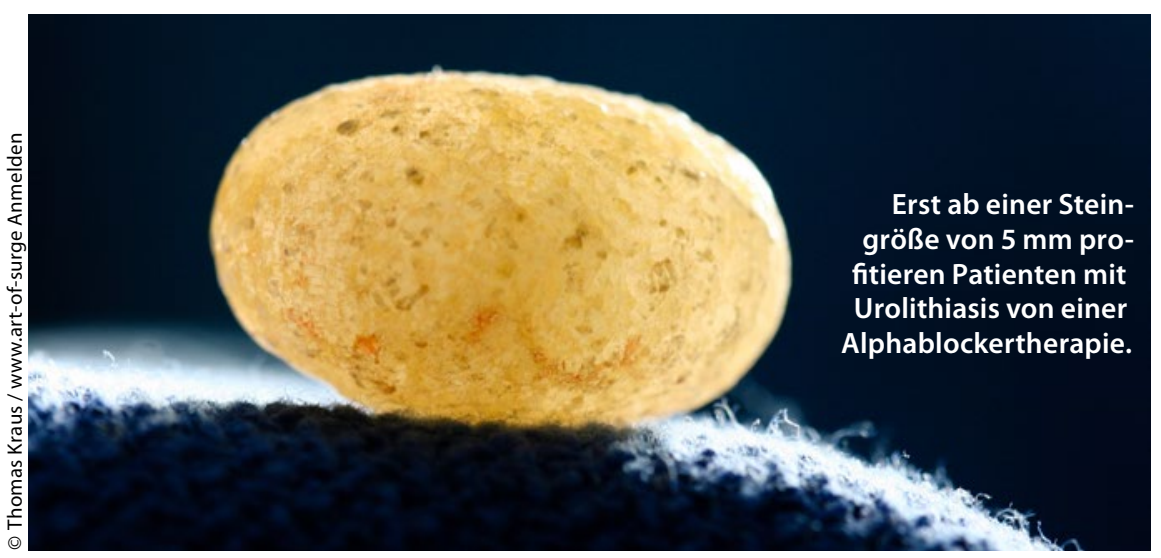

Alphablockertherapie profitiere. Der Anteil der Patienten mit erfolgtem Steinabgang lag in der Interventionsgruppe bei $75,8 \%$ und in der Kontrollgruppe bei $48,2 \%$, wenn eine Nachbeobachtungsdauer von 28 Tagen angelegt wurde. In beiden Gruppen waren die Steine im Mittel 5,7 mm groß.

Bemerkenswerterweise zeigte sich für die Alphablockertherapie kein Behandlungsvorteil für kleinere Steine bis maximal $5 \mathrm{~mm}$, sehr wohl dagegen für größere Konkremente: Für Letztere lag die Wahrscheinlichkeit, dass sie nach der Therapie den Harnleiter passieren konnten, um 57 \% höher als unter Placebo. Ob die Steine im oberen, mittleren oder unteren Drittel des Harnleiters lokalisiert waren, spielte dagegen keine Rolle, ebenso wenig das Geschlecht der Patienten.

Wie die Forscher berichten, mussten sich die medikamentös behandelten Patienten mit deutlich geringerer Wahrscheinlichkeit einer chirurgischen Intervention unterziehen (gepooltes Risikoverhältnis: 0,44). Unter Alphablockertherapie erfolgte zudem die Steinpassage im Mittel um 3,79 Tage früher als in der Placebogruppe. Auch die geringere Zahl der Schmerzepisoden sowie die geringere Häufigkeit der Klinikeinweisungen sprachen für die MET. Unerwünschte Ereignisse waren in beiden Gruppen selten; unter dem Alphablocker erlebten Männer allerdings häufiger eine Ejakulationsstörung.

Fazit: Daten aus früheren Studien hätten gezeigt, so Hollingsworth et al., dass Steine mit einem Durchmesser von höchstens $5 \mathrm{~mm}$ letztlich meist ohne Probleme abgehen. Der zu erwartende Nutzen einer MET bei kleinen Steinen sei daher naturgemäß gering. Die Alphablocker wirkten wahrscheinlich über den Mechanismus einer Relaxation der glatten Muskulatur des Harnleiters. In der Pickard-Studie wurden, so vermuten Hollingsworth et al., mehr Patienten mit kleinen Steinen berücksichtigt, die unter der Placebotherapie spontan abgegangen seien; dies erkläre möglicherweise die unterschiedlichen Ergebnisse.

Dr. Elke Oberhofer

Hollingsworth JM et al. Alpha blockers for treatment of ureteric stones: systematic review and meta-analysis. BMJ. 2016; 355: i6112. 